\title{
Cognitive behavior therapy for psychological distress in patients with recurrent miscarriage
}

This article was published in the following Dove Press journal:

Psychology Research and Behavior Management

18 July 2013

Number of times this article has been viewed

\author{
Yumi Nakano' \\ Tatsuo Akechi ${ }^{2}$ \\ Toshiaki A Furukawa ${ }^{3}$ \\ Mayumi Sugiura-Ogasawara ${ }^{4}$ \\ 'Department of Psychology, School \\ of Human Sciences, Sugiyama \\ Jogakuen University, Nisshin, Aichi, \\ Japan; ${ }^{2}$ Department of Psychiatry \\ and Cognitive-Behavioral Medicine, \\ Nagoya City University, Graduate \\ School of Medical Sciences, Nagoya, \\ Japan; ${ }^{3}$ Department of Health \\ Promotion and Human Behavior \\ (Cognitive-Behavioral Medicine), \\ Kyoto University Graduate School \\ of Medicine, Kyoto, Japan; \\ ${ }^{4}$ Department of Obstetrics and \\ Gynecology, Nagoya City University, \\ Graduate School of Medical Sciences, \\ Nagoya, Japan
}

Objective: To examine the reduction of psychiatric symptoms using individual cognitive behavior therapy (CBT) for women who suffer from recurrent miscarriage (RM) and depression and/or anxiety.

Methods: Patients with RM and a score of five or higher for K6, a self-report screening scale for depression/anxiety, were interviewed to find information about stressful situations, thoughts, and consequent behaviors that are common and potential causes of psychological distress among RM patients. We then performed individual CBT on 14 patients with RM and depression/anxiety, referring to a list from the interviews, and examined the effects of CBT by a paired $t$-test.

Results: Fourteen women received CBT. The mean number of intervention times was 8.9 sessions (standard deviation [SD], 4.6 sessions). The average Beck Depression Inventory-Second Edition and State-Trait Anxiety Inventory-state anxiety scores, self-report screening scales for depression/ anxiety, decreased from $13.6(\mathrm{SD}, 8.2)$ and $49.0(\mathrm{SD}, 7.1)$ at baseline to $5.2(\mathrm{SD}, 4.4)$ and 38.0 (SD, 10.2) posttherapy, respectively. These changes were statistically significant.

Conclusion: The current preliminary open study confirmed that individual CBT was potentially useful for women with RM and depression and/or anxiety. This finding is the first step towards creating a comprehensive psychological support system for women with RM.

Keywords: spontaneous abortion, psychological support, depression, anxiety

\section{Introduction}

Miscarriage is a very common complication in pregnancy, found in $15 \%$ of the women clinically identified as being pregnant. Recurrent miscarriage (RM), defined as two or more consecutive miscarriages, develops in about $5 \%$ of couples. ${ }^{1}$ In recent years, RM has gained social recognition as a problem related to the decreasing birthrate. ${ }^{2}$

Causes of RM include abnormal chromosomes in either partner, existence of antiphospholipid antibodies, and uterine anomalies. Abnormal embryonic karyotype is also known as a cause, accounting for $25 \%-50 \%$ of miscarriages. However, more than half of RM cases remain unexplained. ${ }^{1}$

Miscarriage causes mental distress such as depression, anxiety, anger, and grief. If miscarriage is repeated, mental distress is sustained. ${ }^{3}$ Craig et $\mathrm{al}^{3}$ and Klock et $\mathrm{al}^{4}$ reported that mental disorder was found in two-thirds of RM patients, and Craig et $\mathrm{al}^{3}$ also reported that their anxiety level is similar to that of psychiatric outpatients. All of these suggest that RM patients are a group of people who need appropriate mental care.

In the infertility field, many studies concerning various psychological supports have been conducted for mental distress of patients. Their outcome is divided into two major kinds, improvement of psychological status and improvement of
Correspondence: Yumi Nakano

Department of Psychology, School

of Human Sciences, Sugiyama Jogakuen

University, 3-2005 Takenoyama,

Nissin, Aich 4700136, Japan

Tel +8I 561741452

Fax +8I 561743205

Email nakanotys20I2@sugiyama-u.ac.jp which permits unrestricted noncommercial use, provided the original work is properly cited. 
pregnancy rate. Three meta-analyses lead to a general conclusion that at least one of the two is to be expected..$^{5-7}$

A few previous studies reported that some forms of psychological support including cognitive behavior therapy (CBT) for patients with single miscarriage were useful. ${ }^{8-10}$ In the field of unexplained RM, it has been also indicated that psychological support such as counseling and increased regular checkups in the beginning pregnancy can help raise fertility rate, ${ }^{11-13}$ but no further progress has been made. However, unlike for infertility patients or single-miscarriage patients, little research has been done for RM patients on the effects of psychological support with an outcome of reducing anxiety and depression in daily life.

CBT is one of the psychotherapies that is generally confirmed as being effective in reducing depression and/or anxiety. ${ }^{14}$ In this study, we preliminarily examined the possibility of reducing depression and/or anxiety of the subjects through individual CBT as the first step for constructing a total psychological support system for RM. Generally, in each CBT session, the patient and the therapist utilize specific hardships in the patient's real life to collaboratively identify and examine her perspective (thoughts, recognition) and behavior. They then devise ways to control her distress or find coping strategies for her hardship. Accordingly, having information in advance about the kind of stressful situations, thoughts, and consequent behaviors which are common and potential causes of depression and/or anxiety among RM patients helps the therapist identify a problem to be discussed and in turn smoothly conduct a session. In addition, such information could be used in the course of developing a broadly available psychological support system in the future. Therefore, we first interviewed patients about what situations often cause them distress, and then conducted preliminary individual CBT sessions referring to the previous interview results and observed the change of depression and/or anxiety.

\section{Materials and methods}

\section{Patients}

Subjects were recruited from women who visited the specialized RM outpatient care at Nagoya City University Hospital during the period of April 2008 to September 2010; recruited subjects had a history of two or more consecutive miscarriages and had no children. Two weeks after systematic RM tests and explanation about the results, patients filled in K6, ${ }^{15,16}$ a self-report questionnaire for depression and/or anxiety, and sent it to us by mail. Patients with a score of five or higher on the K6 were selected as subjects in this study.
With those subjects, a psychiatrist (YN) conducted Diagnostic and Statistical Manual of Mental Disorders (fourth edition, text revision) (DSM-IV-TR) diagnosis with Structured Clinical Interview for DSM-IV (SCID), ${ }^{17}$ and the psychiatrist selected those who were diagnosed with mood disorder, anxiety disorder, or adjustment disorder caused by RM-related stress, pregnancy, or childbirth. Exclusion criteria included: (1) serious physical illnesses such as autoimmune disorder, cardiac disease, or chronic respiratory disease; (2) current or past psychotic disorder, bipolar disorder, cyclothymic disorder, eating disorder, developmental disorder, learning disorder, or substance dependence according to DSM-IV-TR; (3) current depression episode, dysthymic disorder, anxiety disorder, or adjustment disorder (by DSM-IV-TR) starting before the first miscarriage; (4) being pregnant at the time of the interview or beginning of CBT; and (5) previous CBT experience. As for new pregnancy during CBT, we planned to make a go/no-go decision when it happened because pregnancy may make some subjects physically (eg, morning sickness) and psychologically (eg, anxiety about miscarriage) unstable.

In general, CBT subjects were instructed to avoid pharmacotherapy. If they were already under pharmacotherapy, they were not allowed to switch medicines during CBT, except in cases of emergency. If a cause of RM was specified, its treatment had priority over CBT.

This study was approved by the Ethics Review Committee at Nagoya City University Graduate School of Medical Sciences. The subjects received explanation in writing and submitted informed consent before entering the study.

\section{Procedure}

Before starting psychological support by CBT, a researcher in this study (YN) with over 15 years of experience in clinical psychiatry and 10 years of experience as a CBT therapist interviewed a series of ten subjects in person, for 75-90 minutes each, and collected information about mental distress concerning pregnancy and children seen in their daily situations. Another psychiatrist (TF) with over 10 years of CBT experience who has been conducting research in psychosocial factors of RM, as well as an obstetrician (MS) who specializes in RM, then examined the interviews. By checking what was said (viewpoints, recognition) and how they behaved in stressful situations, as mentioned by subjects, several features, which seemed helpful in CBT, were extracted (Table 1).

The average age of the ten subjects interviewed was 32.3 $( \pm 3.5)$ years and the average number of miscarriages was 
Table I Common thoughts and behaviors among women with recurrent miscarriage who are experiencing depression and/or anxiety

I. They believe that a woman who marries but does not have a baby is not a mature adult

2. They believe that people who have children are happy and that people who do not have children are unhappy

3. They have feelings of guilt that they have killed their own fetuses in uteri

4. They are afraid of both getting pregnant and of not getting pregnant

5. They are beleaguered with anxiety about the future and about how long their present situation will last

6. They are uncertain if each action in their daily life is good or bad for their next pregnancy

7. They miss many opportunities to enjoy themselves because they avoid places where there are many children and their parents, and participating in events where many mothers will be present

8. They avoid gatherings of relatives during the summer holidays or New Year holidays, or gatherings of peers such as class reunions

9. They and their spouses are likely to overlook women's fatigue and exhaustion

10. Not many women understand that their idea and their husband's idea are not the same regarding having a baby

$2.7( \pm 0.9)$. No significant difference was found between these and the average age of 33.0 (standard deviation $[\mathrm{SD}] \pm 4.8$ ) and the average number of miscarriages of $2.7(\mathrm{SD} \pm 1.0)$ of a series of 305 patients who visited our hospital for thorough examination. The causes of miscarriage for eight subjects were unexplained. Two suffered from uterine deformity, but they did not need surgery in order to become pregnant or to maintain pregnancy. ${ }^{18}$ The average K6 score was $8.6( \pm 2.5)$.
Those who were interviewed to extract common thoughts and behaviors continued regular treatment at the obstetrics department. They also received routine psychiatric outpatient therapy if they needed it.

After common thoughts and behaviors were obtained as indicated in Table 1, 14 subjects who met the eligibility criteria provided informed consent and entered this study between October 2008 and September 2010 (Figure 1).

$\mathrm{N}=255$

First visit for systematic RM tests between

October 2008 and September 2010

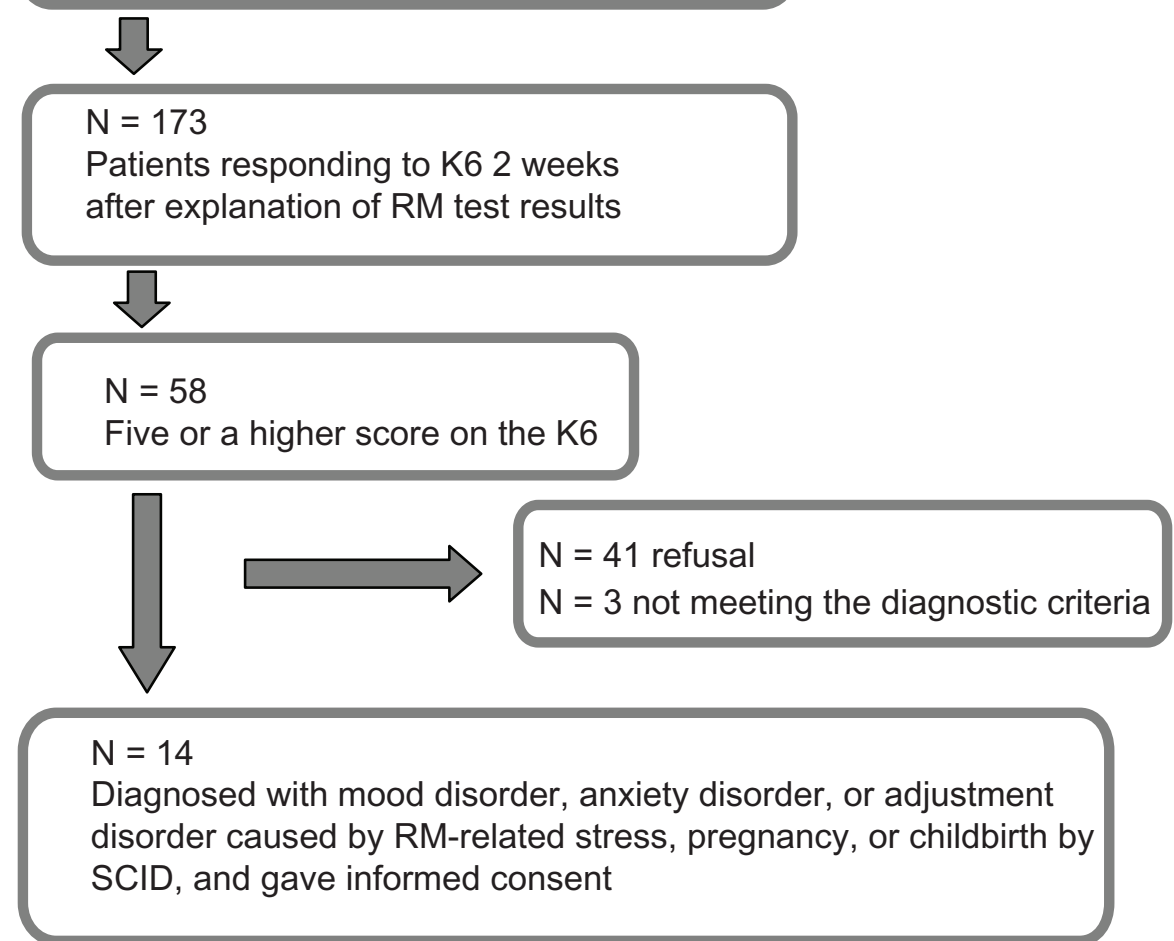

Figure I Patient flow chart for CBT.

Abbreviations: CBT, cognitive behavior therapy; N, number; RM, recurrent miscarriage; SCID, Structured Clinical Interview for the Diagnostic and Statistical Manual of Mental Disorders (fourth edition); K6, 6-item self-report instrument for screening for clinical depression or anxiety in adults. 
The psychiatrist (YN) conducted CBT according to the individual treatment method developed by Beck $^{19}$ as well as by Beck et al (Table 1). ${ }^{20}$ One session lasted approximately 50 minutes. The list in Table 1 was never shown to the patient since it was made as reference to help the therapist conduct smooth and effective sessions.

The basic rule for a course of sessions was that the sessions were to be held once a week, up to 16 times in total. However, in reality, flexible decisions had to be made depending on how profoundly distressed the patient was feeling in her daily life and how much the patient's depression and/or anxiety improved. In some cases, we allowed a patient to have a session once every 2 or more weeks after the 12 th session.

\section{Measure}

\section{K6}

The K6 is a 6-item self-report instrument for screening for clinical depression or anxiety in adults. ${ }^{15}$ The items cover how frequently the respondents experienced symptoms of psychological distress during the previous 30 days. The possible total scores range from 0 to 24 . According to a Japanese validation study of the K6 in a general population, the positive predictive value for major depressive disorder or any anxiety disorder according to the DSM-IV-TR criteria was 0.49 for K6 scores of 10 or more. ${ }^{16}$ The reliability and validity of the Japanese version has been previously confirmed. ${ }^{16}$ The internal consistency of this instrument in the current sample was sufficient (Cronbach's $\alpha=0.89$ ). In this study, women with a score of five or higher on the K6 result were included as subjects.

\section{Beck Depression Inventory-Second Edition}

The Beck Depression Inventory-Second Edition (BDI-II) is a 21-item self-report instrument for measuring the severity of depression in adolescents and adults. ${ }^{21}$ The total scores range from 0 through 63 . The severity of depression is categorized based on the following BDI-II scores in Japanese samples: a score of 13 or less is regarded as minimal (or remission); 14 to 19 is regarded as mild; 20 to 28 is regarded as moderate; and 29 or greater is regarded as severe. The reliability and validity of the Japanese version has been previously confirmed. ${ }^{22}$ The internal consistency of this instrument in the current sample was sufficient (Cronbach's $\alpha=0.92$ ).

\section{State-Trait Anxiety Inventory-state anxiety}

The State-Trait Anxiety Inventory-state (STAI-s) anxiety consists of 20 items and has a maximum score of $80 .{ }^{23}$
State anxiety refers to the degree of anxiety at a particular point. Scores of over 50 indicate an extremely high level of anxiety (neurosis level). The reliability and validity of the Japanese version has been previously confirmed. ${ }^{24}$ The internal consistency of this instrument in the current sample was sufficient (Cronbach's $\alpha=0.91$ ).

BDI-II and STAI-s anxiety were adopted because they are both simple and useful scales to check the change in depression and/or anxiety severity. Following the usual CBT procedure, they were conducted right before each CBT session to assess the patient's condition.

\section{Statistical analysis}

Wilcoxon signed rank tests were conducted before and after CBT to see any statistically-significant change of BDI-II and STAI state anxiety. All analyses were performed using IBM SPSS Statistics, version 18 (IBM Corporation, Armonk, NY, USA). All statistical tests were two-tailed, and an alpha value of less than 0.05 was considered statistically significant.

\section{Results}

Fourteen patients, who met the criteria and consented, received CBT. The features of the samples are shown in Table 2. No significant difference was found between these and a series of 305 patients who visited our hospital for thorough examination in terms of the average age and number

Table 2 Sample characteristics: demographic and symptomatic data

\begin{tabular}{lll}
\hline & Mean & SD \\
\hline Age (years) & 34 & 4 \\
Number of previous miscarriages & 2.7 & 1.0 \\
BDI-Il before CBT & 13.6 & 8.2 \\
State anxiety in STAI before CBT & 49 & 7.1 \\
Number of CBT sessions & 8.9 & 4.6 \\
& $\%$ & Number \\
\hline
\end{tabular}

\section{Education}

High school graduate $\quad 28.6 \quad 4$

Junior college graduate $\quad 50.0 \quad 7$

College graduate or higher $\quad 21.4 \quad 3$

\section{DSM-IV diagnosis}

Major depressive disorder $\quad 35.7 \quad 5$

Adjustment disorder (with depressed mood) $\quad 35.7 \quad 5$

Adjustment disorder (with mixed anxiety $\quad 28.6 \quad 4$

and depressed mood)

Adjustment disorder (with anxiety) $\quad 7.1 \quad$ ।

Specific phobia $\quad 7.1 \quad$ ।

Panic disorder $\quad 7.1 \quad 1$

Posttraumatic stress disorders $7.1 \quad$ ।

Abbreviations: SD, standard deviation; BDI-II, Beck Depression InventorySecond Edition; CBT, cognitive behavior therapy; STAI, The State-Trait Anxiety Inventory; DSM-IV, Diagnostic and Statistical Manual of Mental Disorders (fourth edition). 
of miscarriages. The causes of RM for nine of the patients (64.2\%) were unexplained. Four patients had uterine deformity but their possibility of maintaining pregnancy was not expected to improve much by a surgery. ${ }^{13}$ One patient was diagnosed with hyperthyroidism but her hormone value had been stable within the normal range for over 6 months under pharmacotherapy (desiccated thyroid $75 \mu \mathrm{g}$ ).

Two patients were diagnosed with moderate- to severelevel major depression disorder according to DSM-VI-TR; their BDI-II scores exceeded 30 points at baseline. The depression and/or anxiety severity of most of the patients was around the level of adjustment disorder according to DSM-IV-TR. One patient had been in pharmacotherapy and there was no change in her medication $(75 \mathrm{mg} /$ day sertraline, $1 \mathrm{mg}$ /day lormetazepam) during CBT sessions. There was one patient who became pregnant in the 14 th week of the 16 originally planned sessions. Since she complained of high anxiety about miscarriage and asked for extended treatment, a decision was made to have a session once a month until she reached a stable period, totaling 18 sessions.

Figure 2 shows the changes in BDI-II and STAI-s anxiety before and after CBT. STAI data for four patients were lost. The average BDI-II and STAI-s scores decreased from 13.6 $(\mathrm{SD}, 8.2)$ and $49.0(\mathrm{SD}, 7.1)$ at baseline to $5.2(\mathrm{SD}, 4.4)$ and 38.0 (SD, 10.2) at posttherapy, respectively. These changes were statistically significant (Wilcoxon signed rank test: BDI-II n $=14, \mathrm{z}=-3.2, P=0.001$; STAI-s $\mathrm{n}=10, \mathrm{z}=-2.4$, $P=0.016)$.

\section{Discussion}

This is the first attempt of psychological support with CBT for patients with RM. This study preliminarily confirmed that $\mathrm{CBT}$, in concert with references to the list of common

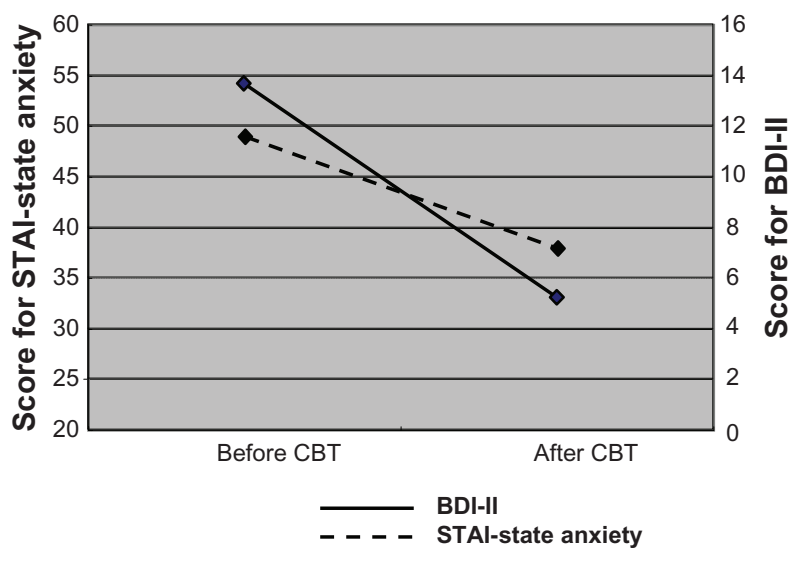

Figure 2 Comparison of BDI-II and STAI before and after CBT.

Abbreviations: BDI-II, Beck Depression Inventory-Second Edition; STAI, StateTrait Anxiety Inventory; CBT, cognitive behavior therapy. hardships prepared in advance, can decrease depression and/or anxiety of patients with RM. It is appropriate to say that, based on the SCID diagnosis and the average BDI-II scores at baseline and posttreatment, the mild depressive state of the 14 subjects in this study recovered to normal levels. It can be also said from the average scores of STAI-s anxiety at baseline and at posttreatment that the subjects came out of their previous condition at a mildly overanxious level.

It has been already mentioned that the childbirth rate of RM patients with an unexplained cause may be raised by increasing the number of checkups and providing counseling in early pregnancy. ${ }^{8-10}$ Additionally, we have already shown that depression raises the possibility of miscarriage. ${ }^{25}$ All these factors indicate that recovery from depression may lead to an increased childbirth rate. Therefore, although we were unable to examine successful birthrate this time, we should, and certainly hope to, have childbirth rate as an outcome in our future research.

However, there are several limitations in this study. First, it was an open label study with no control group. There exists a possibility that depression and/or anxiety decreased as a natural course. Second, it was left unexamined whether the positive effect could be maintained after the end of treatment, despite the fact continuing improvement of depression and/ or anxiety in the life of patients is the most important outcome. Moreover, since there were only two estimating points, one at the start and another at the end of CBT sessions, the process of improvement could not be discussed. The third limitation is that the strict procedure of qualitative research, such as a qualitative and descriptive research method, was not taken in constructing Table 1. However, it was designed as reference data to conduct CBT smoothly in the first place, and in fact, Table 1 fulfilled the purpose. For example, more than half of the subjects covered items $1,3,5,8$, and 10 in their sessions.

We routinely hand out a form to patients to write comments freely when they visit our hospital for thorough examination. Although they often mention experiencing various kinds of psychological distress, only a few patients actually come for individual CBT. As Boivin et $\mathrm{al}^{26}$ pointed out, it is likely that patients with mild psychological distress do not feel a need to depend on a specialist, while those experiencing severe distress do not reach a point where they want to meet with CBT therapists, or any other specialist engaging in psychological support, because these individuals worry about how much these services may cost and what the specialist may be like. Moreover, many patients might naturally be resistant to self-disclosure. 
Recently, there have been many attempts concerning CBT on the Internet. ${ }^{27,28}$ The approach using the Internet may have advantages because it can be accessed from anywhere at low cost, requires less self-disclosure, calls for less concern about compatibility with a therapist, and therefore, seems easier to start. Upon creating online CBT content in the future, the information from Table 1 will contribute considerably.

On the other hand, Wischmann ${ }^{29}$ recommended that face-to-face consultations be used for complicated matters for infertility patients' mental distress. Therefore, for RM patients who are suffering from depression and/or anxiety, we plan to recommend a psychological support program based on CBT via the Web before inviting patients to engage in individual CBT. Once such a system takes off, we hope to conduct a randomized controlled trial with birthrate, as well as depression and/or anxiety, as study endpoints.

This pilot study, which preliminarily indicated a decrease in depression and/or anxiety for RM patients by individual CBT, was the first step towards creating a comprehensive psychological support system for RM.

\section{Acknowledgments}

This study was supported by the Japanese Ministry of Health, Labor, and Welfare, Grant number H20-childrengeneral-002.

\section{Disclosure}

The authors report no conflicts of interest in this work. The authors disclosed no proprietary or commercial interest in any product mentioned or concept discussed in this article.

\section{References}

1. Rai R, Regan L. Recurrent miscarriage. Lancet. 2006;368(9535): 601-611.

2. Ministry of Health Labour and Welfare J. Ministry of Health, Labour and Welfare Annual Report 2011. Tokyo, Japan, 2012. http://www.mhlw. go.jp/toukei/youran/indexyk_1_2.html.

3. Craig M, Tata P, Regan L. Psychiatric morbidity among patients with recurrent miscarriage. J Psychosom Obstet Gynaecol. 2002;23(3): $157-164$.

4. Klock SC, Chang G, Hiley A, Hill J. Psychological distress among women with recurrent spontaneous abortion. Psychosomatics. 1997;38(5): 503-507.

5. Boivin J. A review of psychosocial interventions in infertility. Soc Sci Med. 2003;57(12):2325-2341.

6. de Liz TM, Strauss B. Differential efficacy of group and individual/ couple psychotherapy with infertile patients. Hum Reprod. 2005;20(5): 1324-1332.

7. Hämmerli K, Znoj H, Barth J. The efficacy of psychological interventions for infertile patients: a meta-analysis examining mental health and pregnancy rate. Hum Reprod Update. 2009;15(3):279-295.
8. Nikčević AV. Development and evaluation of a miscarriage follow-up clinic. J Reprod Infant Psychol. 2003;21(3):207-217.

9. Séjourné N, Callahan S, Chabrol H. The utility of a psychological intervention for coping with spontaneous abortion. $J$ Reprod Infant Psychol. 2010;28(3):287-296.

10. Swanson KM. Effects of caring, measurement, and time on miscarriage impact and women's well-being. Nurs Res. 1999;48(6):288-298.

11. Clifford K, Rai R, Regan L. Future pregnancy outcome in unexplained recurrent first trimester miscarriage. Hum Reprod. 1997;12(2): 387-389.

12. Liddell HS, Pattison NS, Zanderigo A. Recurrent miscarriage - outcome after supportive care in early pregnancy. Aust N Z J Obstet Gynaecol. 1991;31(4):320-322.

13. Stray-Pedersen B, Stray-Pedersen S. Etiologic factors and subsequent reproductive performance in 195 couples with a prior history of habitual abortion. Am J Obstet Gynecol. 1984;148(2):140-146.

14. Tolin DF. Is cognitive-behavioral therapy more effective than other therapies? A meta-analytic review. Clin Psychol Rev. 2010;30(6): 710-720.

15. Kessler RC, Andrews G, Colpe LJ, et al. Short screening scales to monitor population prevalences and trends in non-specific psychological distress. Psychol Med. 2002;32(6):959-976.

16. Furukawa TA, Kawakami N, Saitoh M, et al. The performance of the Japanese version of the K6 and K10 in the World Mental Health Survey Japan. Int J Methods Psychiatr Res. 2008;17(3):152-158.

17. First MB, Spitzer RL, Gibbon M, Williams JBW. User's Guide for the Structured Clinical Interview for DSM-IV Axis I Disorders: SCID-1 Clinician Version. Arlington, VA: American Psychiatric Press; 1997.

18. Sugiura-Ogasawara M, Ozaki Y, Kitaori T, Kumagai K, Suzuki S. Midline uterine defect size is correlated with miscarriage of euploid embryos in recurrent cases. Fertil Steril. 2010;93(6):1983-1988.

19. Beck AT. Cognitive Therapy of Depression. New York, NY: Guilford Press; 1979 .

20. Beck AT, Emery G, Greenberg R. Anxiety Disorders and Phobias: A Cognitive Perspective. New York, NY: Basic Books; 1985.

21. Beck AT, Steer RA, Brown GK. Manual for the Beck Depression Inventory-II. San Antonio, TX: The Psychological Corporation; 1996.

22. Hiroe T, Kojima M, Yamamoto I, et al. Gradations of clinical severity and sensitivity to change assessed with the Beck Depression Inventory-II in Japanese patients with depression. Psychiatry Res. 2005;135(3):229-235.

23. Spielberger CD, Gorsuch RL, Lushene R. Manual for the State-Trait Anxiety Inventory, ConsultingPsychologists press, california,1973.

24. Iwata N, Mishima N, Okabe K, Kobayashi N, Hashiguchi E, Egashira K. Psychometric properties of the State-Trait Anxiety Inventory among Japanese clinical outpatients. J Clin Psychol. 2000;56(6): 793-806.

25. Nakano Y, Oshima M, Sugiura-Ogasawara M, Aoki K, Kitamura T, Furukawa TA. Psychosocial predictors of successful delivery after unexplained recurrent spontaneous abortions: a cohort study. Acta Psychiatr Scand. 2004;109(6):440-446.

26. Boivin J, Scanlan LC, Walker SM. Why are infertile patients not using psychosocial counselling? Hum Reprod. 1999;14(5):1384-1391.

27. Lewis C, Pearce J, Bisson JI. Efficacy, cost-effectiveness and acceptability of self-help interventions for anxiety disorders: systematic review. Br J Psychiatry. 2012;200(1):15-21.

28. Reger MA, Gahm GA. A meta-analysis of the effects of internet- and computer-based cognitive-behavioral treatments for anxiety. J Clin Psychol. 2009;65(1):53-75.

29. Wischmann T. Implications of psychosocial support in infertility - a critical appraisal. J Psychosom Obstet Gynaecol. 2008;29(2):83-90. 
Psychology Research and Behavior Management

Dovepress

\section{Publish your work in this journal}

Psychology Research and Behavior Management is an international, peerreviewed, open access journal focusing on the science of psychology and its application in behavior management to develop improved outcomes in the clinical, educational, sports and business arenas. Specific topics covered include: Neuroscience, memory \& decision making; Behavior modification \& management; Clinical applications; Business \& sports performance management; Social and developmental studies; Animal studies. The manuscript management system is completely online and includes a quick and fair peer-review system. Visit http://www.dovepress. com/testimonials.php to read real quotes from published authors.

Submit your manuscript here: http://www.dovepress.com/psychology-research-and-behavior-management-journal 